J. Nonlinear Var. Anal. 2 (2018), No. 1, pp. 35-47

Available online at http://jnva.biemdas.com

https://doi.org/10.23952/jnva.2.2018.1.04

\title{
A REMARK ON THE FIXED POINT PROPERTY OF NONEXPANSIVE MAPPINGS
}

\author{
MONIKA BUDZYŃSKA ${ }^{1}$, ALEKSANDRA GRZESIK ${ }^{2}$, WIESŁAWA KACZOR ${ }^{1}$, TADEUSZ KUCZUMOW ${ }^{1, *}$ \\ ${ }^{1}$ Instytut Matematyki, UMCS, 20-031 Lublin, Poland \\ ${ }^{2}$ Wydziat Matematyki i Fizyki Stosowanej, Politechnika Rzeszowska, 35-959 Rzeszów, Poland
}

\begin{abstract}
The following problem is studied in this paper. Assume that $(X,\|\cdot\|)$ is a Banach space with an unconditional Schauder basis. Does it admit an equivalent norm so that the renormed space has the fixed point property but fails normal structure?
\end{abstract}

Keywords. Fixed point; Kadec-Klee property; Nonexpansive mapping; Normal structure; Unconditional basis.

2010 Mathematics Subject Classification. 47H10.

\section{INTRODUCTION}

In this paper, we study the following open problem. Assume that $(X,\|\cdot\|)$ is a separable Banach space. It is well known that it can be equivalently renormed in such a way that $X$ with the new norm has normal structure ([29] and also [11]) and therefore by the famous Kirk theorem ([17]) it has the fixed point property for nonexpansive self-mappings acting on weakly compact and convex subsets of $X$. So it is natural to ask whether we can equivalently renorm a Banach space with an unconditional Schauder basis so that the renormed space has the fixed point property but has a "bad" geometrical property; namely, it fails normal structure. Our research is motivated by the Karlovitz result about renorming of the Hilbert space $l^{2}$ ([16]) and the Lin results about the Banach spaces with the 1-unconditional Schauder bases ([19]). Here we need to mention that T. Domínguez Benavides proved that every reflexive Banach space can be renormed to satisfy the fixed point property for nonexpansive mappings [10].

\section{BASIC NOTIONS AND FACTS}

We first recall a few notions and facts from geometry of Banach spaces. Throughout this paper all Banach spaces are infinite dimensional and real. We begin this section with the following well known definitions and results ([1], [2], [7], [8], [9], [11], [12], [13], [14], [15], [17], [18], [19], [20], [21], [24] and [26]).

Definition 2.1. Let $(X,\|\cdot\|)$ be a Banach space. A sequence $\left\{e_{i}\right\}_{i}$ in $X$ is called a Schauder basis of $X$ if for each $x \in X$ there exists a unique sequence of scalars $\left\{x^{i}\right\}_{i}$ such that $x=\sum_{i=1}^{\infty} x^{i} e_{i}$. A basis $\left\{e_{i}\right\}_{i}$ is said to be normalized if $\left\|e_{i}\right\|=1$ for all $i$.

\footnotetext{
${ }^{*}$ Corresponding author.

E-mail addresses: monikab1@ hektor.umcs.lublin.pl (M. Budzyńska), a.grzesik22@gmail.com (A. Grzesik), wkaczor@he ktor.umcs.lublin.pl (W. Kaczor), tadek@ hektor.umcs.lublin.pl (T. Kuczumow).

Received October 11, 2017; Accepted November 13, 2017.
}

(C)2018 Journal of Nonlinear and Variational Analysis 
Definition 2.2. Let $(X,\|\cdot\|)$ be a Banach space with a Schauder basis $\left\{e_{i}\right\}_{i}$. For every integer $j$ a linear functional $e_{j}^{*}$ is defined by $e_{j}^{*}\left(\sum_{i=1}^{\infty} x^{i} e_{i}\right)=x^{j}$. These functionals $e_{i}^{*}, i=1,2, \ldots$, are called biorthogonal functionals associated with the basis $\left\{e_{i}\right\}_{i}$.

Remark 2.1. Throughout this paper we assume that for each considered Schauder basis $\left\{e_{i}\right\}_{i}$ there exist constants $0<\tilde{m} \leq \tilde{M}<\infty$ such that $\tilde{m} \leq\left\|e_{i}\right\| \leq \tilde{M}$ for each $i \in \mathbb{N}$. Then for the biorthogonal functionals $\left\{e_{i}^{*}\right\}_{i}$ associated with this Schauder basis $\left\{e_{i}\right\}_{i}$ there also exist constants $0<\tilde{m}_{1} \leq \tilde{M}_{1}<\infty$ such that $\tilde{m}_{1} \leq\left\|e_{i}^{*}\right\| \leq \tilde{M}_{1}$ for each $i \in \mathbb{N}$. Additionally we have $\lim _{i} e_{i}^{*}(x)=0$ for each $x \in X$.

Definition 2.3. Let $(X,\|\cdot\|)$ be a Banach space with a Schauder basis $\left\{e_{i}\right\}_{i}$. We say that the Schauder basis $\left\{e_{i}\right\}_{i}$ is unconditional if whenever the series $\sum_{i=1}^{\infty} x^{i} e_{i}$ converges, it converges unconditionally, i.e., $\sum_{i=1}^{\infty} x^{\sigma(i)} e_{\sigma(i)}$ converges for every permutation $\sigma$ of $\mathbb{N}$.

Some equivalent definitions of unconditionality of a Schauder basis are presented below.

Theorem 2.1. Let $(X,\|\cdot\|)$ be a Banach space with a Schauder basis $\left\{e_{i}\right\}_{i}$. The following statements are equivalent:

(1) the basis $\left\{e_{i}\right\}_{i}$ is unconditional;

(2) for every choice of signs $\left\{\varepsilon_{i}\right\}_{i}$ (i.e., $\left.\varepsilon_{i}= \pm 1\right) \sum_{i=1}^{\infty} \varepsilon_{i} x^{i} e_{i}$ converges whenever $\sum_{i=1}^{\infty} x^{i} e_{i}$ converges;

(3) for every convergent series $\sum_{i=1}^{\infty} x^{i} e_{i}$ and for every sequence of scalars $\left\{b^{i}\right\}_{i}$ such that $\left|b^{i}\right| \leq\left|x^{i}\right|$ for all $i$ the series $\sum_{i=1}^{\infty} b^{i} e_{i}$ converges.

Let us recall also that if $\left\{e_{i}\right\}_{i}$ is an unconditional basis in a Banach space $(X,\|\cdot\|)$, then

$$
\sup \left\{\left\|\sum_{i=1}^{\infty} \varepsilon_{i} x^{i} e_{i}\right\|:\left\|\sum_{i=1}^{\infty} x^{i} e_{i}\right\|=1 \text { and } \varepsilon_{i}= \pm 1\right\}
$$

is finite. The following concept is useful in studying properties of Banach spaces.

Definition 2.4. If a Schauder basis $\left\{e_{i}\right\}_{i}$ is unconditional, then the number

$$
K:=\sup \left\{\left\|\sum_{i=1}^{\infty} \varepsilon_{i} x^{i} e_{i}\right\|:\left\|\sum_{i=1}^{\infty} x^{i} e_{i}\right\|=1 \text { and } \varepsilon_{i}= \pm 1\right\}
$$

is called the unconditional constant of $\left\{e_{i}\right\}_{i}$. If this constant $K$ is equal to 1 , then we say that $\left\{e_{i}\right\}_{i}$ is a 1-unconditional basis.

Since in our paper all Banach spaces are real, we have

Theorem 2.2. If a Schauder basis $\left\{e_{i}\right\}_{i}$ is unconditional, then the number

$$
\sup \left\{\left\|\sum_{i=1}^{\infty} s_{i} x^{i} e_{i}\right\|:\left\|\sum_{i=1}^{\infty} x^{i} e_{i}\right\|=1 \text { and }\left|s_{i}\right| \leq 1\right\}
$$

is equal to the unconditional constant of $\left\{e_{i}\right\}_{i}$.

Definition 2.5. If a Schauder basis $\left\{e_{i}\right\}_{i}$ is unconditional and $F$ is a nonempty subset of $\mathbb{N}$, then the projection

$$
P\left(\sum_{i=1}^{\infty} x^{i} e_{i}\right):=\sum_{i \in F} x^{i} e_{i}
$$

is called the natural projection associated with $F$ and the unconditional basis $\left\{e_{i}\right\}_{i}$. 
Remark 2.2. By Theorem 2.2, the norm of any natural projection does not exceed the unconditional constant of the basis.

Remark 2.3. Observe that if $\left\{e_{i}\right\}_{i}$ is 1-unconditional, then for each $x=\sum_{i=1}^{\infty} e_{i}^{*}(x) e_{i} \in X$ and for each choice of signs $\left\{\varepsilon_{i}\right\}_{i}$ we have

$$
\|x\|=\left\|\sum_{i=1}^{\infty} e_{i}^{*}(x) e_{i}\right\|=\left\|\sum_{i=1}^{\infty} \varepsilon_{i} e_{i}^{*}(x) e_{i}\right\| .
$$

Remark 2.4. Recall that if a Schauder basis $\left\{e_{i}\right\}_{i}$ in a Banach space $(X,\|\cdot\|)$ is 1-unconditional, then we can replace it by the Schauder basis $\left\{e_{i, 1}\right\}_{i}=\left\{\alpha_{i} e_{i}\right\}_{i}$ with real $\alpha_{i}$ for $i \in \mathbb{N}$ and this new basis $\left\{e_{i, 1}\right\}_{i}$ is still 1-unconditional in $(X,\|\cdot\|)$.

Remark 2.5. It is known that each Banach space $(X,\|\cdot\|)$ with an unconditional Schauder basis $\left\{e_{i}\right\}_{i}$ can be equivalently renormed in such a way that the basis $\left\{e_{i}\right\}_{i}$ is 1-unconditional in the new norm. To this end we simply set

$$
\|x\|_{1}:=\sup \left\{\left\|\sum_{i=1}^{\infty} \beta_{i} e_{i}^{*}(x) e_{i}\right\|: \sup _{i}\left|\beta_{i}\right| \leq 1\right\} .
$$

Then we have (see Remark 2.2)

$$
\|x\|_{1}:=\sup _{n=0,1,2, \ldots}\left\|x-P_{n} x\right\|_{1}
$$

where $\left\{P_{n}\right\}_{n=0}^{\infty}$ is a sequence of the standard projections in $X$ which are defined in the following way: $P_{0}:=0$ and $P_{n} x:=\sum_{i=1}^{n} e_{i}^{*}(x) e_{i}$ for $n=1,2, \ldots$ and $x \in X$. Observe that the sequence of operator norms of projections $P_{n}, n=1,2, \ldots$ with respect to the norm $\|\cdot\|\left(\|\cdot\|_{1}\right)$ in $X$ is bounded.

Now we recall definitions of a few geometric properties of Banach spaces. We begin with the definition of the non-strict Opial property.

Definition 2.6. A Banach space $(X,\|\cdot\|)$ has the non-strict Opial property if for each weakly null sequence $\left\{x_{n}\right\}_{n}$ and each $x$ in $X$ we have

$$
\limsup _{n}\left\|x_{n}\right\| \leq \limsup _{n}\left\|x_{n}-x\right\| .
$$

Now we recall the notion of local uniform rotundity.

Definition 2.7. We say that a Banach space $(X,\|\cdot\|)$ is locally uniformly rotund (LUR) if for each $x \in X$ every sequence $\left\{x_{n}\right\}_{n}$ with $\lim _{n}\left\|x_{n}\right\|=\|x\|$ and $\lim _{n}\left\|x+x_{n}\right\|=2\|x\|$ tends strongly to $x$.

We will also use the Kadec-Klee property of a Banach space.

Definition 2.8. Let $(X,\|\cdot\|)$ be a Banach space. We say that $(X,\|\cdot\|)$ has the Kadec-Klee property with respect to the weak topology (simply the Kadec-Klee property) if each sequence $\left\{x_{n}\right\}_{n}$ with $\lim _{n}\left\|x_{n}\right\|=$ 1 , which converges weakly to a point $x$ with $\|x\|=1$, tends strongly to $x$.

The connection between the last two properties of Banach spaces is shown in the following theorem.

Theorem 2.3. Let $(X,\|\cdot\|)$ be a Banach space. If $(X,\|\cdot\|)$ is locally uniformly rotund, then $(X,\|\cdot\|)$ has the Kadec-Klee property with respect to the weak topology.

In our considerations the notion of universality will play an important role. 
Definition 2.9. A Banach space $\left(Y,\|\cdot\|_{Y}\right)$ is universal for a class of separable Banach spaces if every separable Banach space $\left(X,\|\cdot\|_{X}\right)$ is congruent to a subspace $Y_{1}$ of $Y$, i.e., there exists a linear and norm-preserving isomorphism $T: X \rightarrow Y_{1}, T(X)=Y_{1}$.

The following result is crucial to our purpose.

Theorem 2.4. The space $l^{\infty}$ of all bounded real sequences furnished with the standard sup-norm is universal for the class of separable Banach spaces.

Remark 2.6. If $\left(X,\|\cdot\|_{X}\right)$ and $\left(Y,\|\cdot\|_{Y}\right)$ are Banach spaces and $T: X \rightarrow Y$ is linear isometric embedding of $X$ into $Y$, then we identify $T(X)$ with $X$ and write $X \subset Y$.

Now we give a few definitions and results from metric fixed point theory.

Definition 2.10. Let $(X,\|\cdot\|)$ be a Banach space and let $C$ be a weakly compact and convex subset of $X$. We say that $C$ has the fixed point property if each nonexpansive mapping $T: C \rightarrow C$ (i.e., $\left\|T x_{1}-T x_{2}\right\| \leq$ $\left\|x_{1}-x_{2}\right\|$ for every $x_{1}, x_{2} \in C$ ) has a fixed point.

If each weakly compact and convex subset $C$ of $X$ has the fixed point property then we say that a Banach space $(X,\|\cdot\|)$ has the fixed point property.

Definition 2.11. Let $(X,\|\cdot\|)$ be a Banach space and let $C$ be a nonempty, non-singleton, bounded and convex subset of $X$. If any convex subset $C_{1}$ of $C$ which contains more than one point has a nondiametral point, i.e., there exists a point $\tilde{x} \in C_{1}$ such that

$$
\sup \left\{\|x-\tilde{x}\|: x \in C_{1}\right\}<\operatorname{diam}_{\|\cdot\|}\left(C_{1}\right)=\sup \left\{\left\|x^{\prime}-x^{\prime \prime}\right\|: x^{\prime}, x^{\prime \prime} \in C_{1}\right\},
$$

then we say that the set $\mathrm{C}$ has normal structure.

We say that a Banach space $(X,\|\cdot\|)$ has normal structure if each nonempty, non-singleton, bounded and convex set $C \subset X$ has normal structure.

Definition 2.12. Let $(X,\|\cdot\|)$ be a Banach space. If for each nonempty, non-singleton, bounded and convex set $C \subset X$ and for each sequence $\left\{x_{i}\right\}_{i}$ in $C$ satisfying $x_{i}-x_{i+1} \rightarrow 0$ as $i \rightarrow \infty$, there exists a point $\tilde{x} \in C$ such that $\liminf _{i}\left\|x_{i}-\tilde{x}\right\|<\operatorname{diam}_{\|\cdot\|}(\mathrm{C})$, then we say that a Banach space $(X,\|\cdot\|)$ has asymptotic normal structure.

Observe that the concepts of normal structure and asymptotic normal structure were effectively used in fixed point theorems of nonexpansive mappings. Namely, in 1965 W. A. Kirk published his famous fixed point theorem.

Theorem 2.5. Let $C$ be a nonempty, weakly compact, convex subset of a Banach space $(X,\|\cdot\|)$. Suppose that $C$ has normal structure. Then each nonexpansive mapping $T: C \rightarrow C$ has a fixed point.

Later in $1976 \mathrm{~L}$. A. Karlovitz proved that the space $X_{J}=X_{\frac{1}{\sqrt{2}}}$, i.e. the space $l^{2}$ renormed according to

$$
\|x\|_{\frac{1}{\sqrt{2}}}:=\max \left\{\|x\|_{\infty}, \frac{\|x\|_{2}}{\sqrt{2}}\right\}
$$

where $\|\cdot\|_{\infty}$ and $\|\cdot\|_{2}$ are usual norms in $l^{\infty}$ and $l^{2}$, respectively, lacks normal structure but has the fixed point property. As J. B. Baillon and R. Schöneberg observed the space $X_{\frac{1}{\sqrt{2}}}$ has in fact asymptotic normal structure and the Karlovitz result is a corollary from the following theorem. 
Theorem 2.6. Every reflexive Banach space with asymptotic normal structure has the fixed point property for nonexpansive mappings.

But in our paper the following fixed point theorem, which is due to P.-K. Lin, plays a crucial role.

Theorem 2.7. Any Banach space with a 1-unconditional Schauder basis has the fixed point property.

Observe that as a consequence of this theorem each Banach space $X_{\beta}=\left(l^{2},\|x\|_{\beta}\right)$, where $0<\beta<1$ and

$$
\|x\|_{\beta}:=\max \left\{\|x\|_{\infty}, \beta\|x\|_{2}\right\}
$$

has the fixed point property.

\section{UNCONDITIONALITY AND 1-UNCONDITIONALITY OF BASES, THE NON-STRICT OPIAL PROPERTY AND THE KADEC-KLEE PROPERTY}

We begin with the following auxiliary theorem.

Theorem 3.1 ([5], see also [11]). Let $(X,\|\cdot\|)$ be a Banach space with a Schauder basis $\left\{e_{i}\right\}_{i}$ and let $\left\{P_{n}\right\}_{n \geq 0}$ be a sequence of projections associated with this basis, i.e., $P_{0} \equiv 0$ and $P_{k} x=\sum_{i=1}^{k} e_{i}^{*}(x) e_{i}$ for $x \in X$ and $k=1,2, \ldots$. Then the norm $\|\cdot\|_{1}$ in $X$, defined by

$$
\|x\|_{1}=\sup _{k=0,1, \ldots}\left\|x-P_{k} x\right\|
$$

for $x \in X$, is equivalent to the norm $\|\cdot\|$ and the Banach space $\left(X,\|\cdot\|_{1}\right)$ has the nonstrict Opial property.

Directly from this theorem we arrive at

Theorem 3.2. Each Banach space with a 1-unconditional Schauder basis has the non-strict Opial property.

Proof. It follows from Theorem 2.2 and Remark 2.2 that for each $x \in X$ we have

$$
\|x\|=\sup _{k=0,1, \ldots}\left\|x-P_{k} x\right\|,
$$

where $P_{0} \equiv 0$ and $P_{k} x=\sum_{i=1}^{k} e_{i}^{*}(x) e_{i}$ for $k=1,2, \ldots$. Therefore by Theorem 3.1 the Banach space $(X,\|\cdot\|)$ has the non-strict Opial property.

Hence the following theorem is valid.

Theorem 3.3. Each Banach space $(X,\|\cdot\|)$ with an unconditional Schauder basis $\left\{e_{i}\right\}_{i}$ can be renormed in such a way that the basis $\left\{e_{i}\right\}_{i}$ is 1-unconditional in the new norm $\|\cdot\|_{1}$ and $\left(X,\|\cdot\|_{1}\right)$ has the non-strict Opial property.

Now we recall the Kadec-Klee theorem.

Theorem $3.4([15],[18])$. Let $\left(Y,\|\cdot\|_{Y}\right)$ be an infinite dimensional Banach space, $Y^{*}$ be the dual space of $Y$ with the standard norm $\|\cdot\|_{Y^{*}}$, and let $X$ be a closed separable subspace in $\left(Y^{*},\|\cdot\|_{Y^{*}}\right)$. Then there exists an equivalent norm $\|\cdot\|_{Y, 1}$ in $Y$ and a norm $\|\cdot\|_{Y^{*}, 1}$ in $Y^{*}$ associated with $\|\cdot\|_{Y, 1}$ such that if $\left\{y_{i}\right\}$ is any sequence in $Y^{*}$ which converges weakly* to $\tilde{y} \in X$, and if $\lim _{j}\left\|y_{j}\right\|_{Y^{*}, 1}=\|\tilde{y}\|_{Y^{*}, 1}$, then $\lim _{j}\left\|y_{j}-\tilde{y}\right\|_{Y^{*}}=0$. 
If additionally the infinite dimensional Banach subspace $X$ of $\left(Y,\|\cdot\|_{Y}\right)$ has a 1-unconditional Schauder basis then we obtain the following modification of the above theorem.

Theorem 3.5. Let $\left(Y,\|\cdot\|_{Y}\right)$ be an infinite dimensional Banach space, $Y^{*}$ be the dual space of $Y$ with the standard norm $\|\cdot\|_{Y^{*}}$, and let $X$ be a closed separable subspace in $\left(Y^{*},\|\cdot\|_{Y^{*}}\right)$. Assume that $X$ has a Schauder basis $\left\{e_{i}\right\}_{i}$ which is 1-unconditional in the norm $\|\cdot\|_{Y^{*}}$. Then there exists an equivalent norm $\|\cdot\|_{Y, 1}$ in $Y$ and a norm $\|\cdot\|_{Y^{*}, 1}$ in $Y^{*}$ associated with $\|\cdot\|_{Y, 1}$ such that

(1) the Schauder basis $\left\{e_{i}\right\}_{i}$ in $X$ is 1 -unconditional in the norm $\|\cdot\|_{Y^{*}, 1}$,

(2) if $\left\{y_{i}\right\}$ is any sequence in $Y^{*}$ which converges weakly* to $\tilde{y} \in X$, and if $\lim _{j}\left\|y_{j}\right\|_{Y^{*}, 1}=\|\tilde{y}\|_{Y^{*}, 1}$, then $\lim \left\|y_{j}-\tilde{y}\right\|_{Y^{*}}=0$.

Proof. Our proof is a modification of the proof given in [7] (see also [14] and [20]). Let $X_{n}=\operatorname{lin}\left\{e_{1}, \ldots, e_{n}\right\}$ for $n=1,2, \ldots$. We define a new norm $\|\cdot\|_{Y^{*}, 1}$ in $Y^{*}$ by setting

$$
\left\|y^{*}\right\|_{Y^{*}, 1}:=\left\|y^{*}\right\|_{Y^{*}}+\sum_{n=1}^{\infty} \frac{1}{2^{n}} \operatorname{dist}_{\|\cdot\|_{Y^{*}}}\left(y^{*}, X_{n}\right)
$$

for each $y^{*} \in Y^{*}$, where dist $\|_{\|\cdot\|_{Y^{*}}}\left(y^{*}, X_{n}\right)$ denotes the distance with respect to the norm $\|\cdot\|_{Y^{*}}$ of $y^{*}$ from $X_{n}$. Observe that by 1 -unconditionality of the Schauder basis $\left\{e_{i}\right\}_{i}$ in the norm $\|\cdot\|_{Y^{*}}$ we have

$$
\|x\|_{Y^{*}}=\left\|\sum_{i=1}^{\infty} e_{i}^{*}(x) e_{i}\right\|_{Y^{*}}=\left\|\sum_{i=1}^{\infty} \varepsilon_{i} e_{i}^{*}(x) e_{i}\right\|_{Y^{*}}
$$

and

$$
\operatorname{dist}_{\|\cdot\|_{Y^{*}}}\left(x, X_{n}\right)=\operatorname{dist}_{\|\cdot\|_{Y^{*}}}\left(\sum_{i=1}^{\infty} e_{i}^{*}(x) e_{i}, X_{n}\right)=\operatorname{dist}_{\|\cdot\|_{Y^{*}}}\left(\sum_{i=1}^{\infty} \varepsilon_{i} e_{i}^{*}(x) e_{i}, X_{n}\right)
$$

for $x=\sum_{i=1}^{\infty} e_{i}^{*}(x) e_{i} \in X$ and for every choice of signs $\left\{\varepsilon_{i}\right\}_{i}$ (i.e., $\varepsilon_{i}= \pm 1$ ) and $n=1,2, \ldots$ This implies that

$$
\|x\|_{Y^{*}, 1}=\left\|\sum_{i=1}^{\infty} e_{i}^{*}(x) e_{i}\right\|_{Y^{*}, 1}=\left\|\sum_{i=1}^{\infty} \varepsilon_{i} e_{i}^{*}(x) e_{i}\right\|_{Y^{*}, 1}
$$

for each $x=\sum_{i=1}^{\infty} e_{i}^{*}(x) e_{i} \in X$ and every choice of signs $\left\{\varepsilon_{i}\right\}_{i}$. Therefore by Definition 2.4 we get that the Schauder basis $\left\{e_{i}\right\}_{i}$ in $X$ is 1-unconditional in the norm $\|\cdot\|_{Y, 1}$. The rest of the proof runs as in [7] (see also [20], the proof of Proposition 1.b.11).

We also have the following theorem ([5]) which proof is partially based on ideas given in [11].

Theorem 3.6. For every infinite dimensional Banach space $\left(X,\|\cdot\|_{X}\right)$ with a Schauder basis $\left\{e_{i}\right\}_{i}$ there exists an equivalent norm $\|\cdot\|_{X, 1}$, such that $\left(X,\|\cdot\|_{X, 1}\right)$ has the Kadec-Klee property and the non-strict Opial property. If additionally the Schauder basis $\left\{e_{i}\right\}_{i}$ is unconditional, then we can construct the norm $\|\cdot\|_{X, 1}$ in such a way that this basis $\left\{e_{i}\right\}_{i}$ is 1-unconditional in the new norm $\|\cdot\|_{X, 1}$.

Proof. The first part of this theorem was proved in [5], but for the convenience of the reader we recall it. So we start with the observation that by Theorem 2.4 we have $X \subset l^{\infty}=\left(l^{1}\right)^{*}=Y^{*}$ with $Y=l^{1}$. It follows from Theorem 3.5 that in the Banach space $l^{\infty}$ with the standard norm $\|\cdot\|_{\infty}$ there exists an equivalent norm $\|\cdot\|_{1}$ such that if $\left\{y_{i}\right\}$ is any sequence in $l^{\infty}$ that converges weakly* to $\tilde{y} \in X$, and if $\lim _{i}\left\|y_{i}\right\|_{1}=\|\tilde{y}\|_{1}$, then $\lim _{i}\left\|y_{i}-\tilde{y}\right\|_{\infty}=0$. Let $\left\{e_{i}\right\}_{i}$ be a Schauder basis in $\left(X,\|\cdot\|_{X}\right)$ and let $\left\{e_{i}^{*}\right\}_{i}$ be a sequence of biorthogonal functionals associated with the basis $\left\{e_{i}\right\}_{i}$. Let $\left\{P_{n}\right\}_{n=0}^{\infty}$ denote a sequence of the standard projections in $X$ which are defined in the following way: $P_{0}:=0$ and $P_{n} x:=\sum_{i=1}^{n} e_{i}^{*}(x) e_{i}$ for 
$n=1,2, \ldots$ and $x \in X$. As we know the sequence $\left\{\left\|P_{n}\right\|_{1}\right\}_{n=0}^{\infty}$ of operator norms (with respect to the norm $\|\cdot\|_{1}$ in $X$ ) of projections is bounded (Remark 2.5). Thus we can introduce a new norm in $X$ as follows

$$
\|x\|_{X, 1}:=\sup _{n=0,1,2, \ldots}\left\|x-P_{n} x\right\|_{1} .
$$

The norm $\|\cdot\|_{X, 1}$ is equivalent to both the norms $\|\cdot\|_{X}$ and $\|\cdot\|_{1}$ in $X$. Moreover, the operator norm $\left\|I-P_{n}\right\|_{X, 1}$ with respect to the norm $\|\cdot\|_{X, 1}$ in $X$ is equal to 1 . Consequently, by Theorem 3.1 the Banach space $\left(X,\|\cdot\|_{X, 1}\right)$ has the non-strict Opial property.

We claim that the Banach space $\left(X,\|\cdot\|_{X, 1}\right)$ has the Kadec-Klee property. Indeed, if the sequence $\left\{x_{i}\right\}_{i}$ in $X$ tends weakly to $\tilde{x} \in X$ and $\lim _{i}\left\|x_{i}\right\|_{X, 1}=\|\tilde{x}\|_{X, 1}=1$, then we have

$$
1=\|\tilde{x}\|_{X, 1}=\left\|\tilde{x}-P_{\bar{n}} \tilde{x}\right\|_{1}
$$

for some $0 \leq \bar{n}<\infty$ since $\lim _{n} P_{n} \tilde{x}=\tilde{x}$. Next $\lim _{i} P_{\bar{n}} x_{i}=P_{\bar{n}} \tilde{x}$ and therefore the sequence $\left\{x_{i}-P_{\bar{n}} x_{i}\right\}_{i}$ tends weakly in $X$ to $\tilde{x}-P_{\bar{n}} \tilde{x}$. Hence

$$
\begin{gathered}
1=\|\tilde{x}\|_{X, 1}=\left\|\tilde{x}-P_{\bar{n}} \tilde{x}\right\|_{1} \leq \liminf _{i}\left\|x_{i}-P_{\bar{n}} x_{i}\right\|_{1} \\
\leq \underset{i}{\limsup }\left\|x_{i}-P_{\bar{n}} x_{i}\right\|_{1} \leq \underset{i}{\limsup }\left\|x_{i}\right\|_{X, 1}=1
\end{gathered}
$$

and this means that

$$
\lim _{i}\left\|x_{i}-P_{\bar{n}} x_{i}\right\|_{1}=\left\|\tilde{x}-P_{\bar{n}} \tilde{x}\right\|_{1}=1
$$

But this implies

$$
0=\lim _{i}\left\|\left(x_{i}-P_{\bar{n}} x_{i}\right)-\left(\tilde{x}-P_{\bar{n}} \tilde{x}\right)\right\|_{\infty}=\lim _{i}\left\|x_{i}-\tilde{x}\right\|_{\infty}=\lim _{i}\left\|x_{i}-\tilde{x}\right\|_{1}
$$

and hence we have the claimed $\operatorname{limit}_{i} \lim _{i}=\tilde{x}$ in $\left(X,\|\cdot\|_{X, 1}\right)$, i.e., the Kadec-Klee property is proved.

In order to prove the second part of our theorem it is enough to observe that, if the Schauder basis $\left\{e_{i}\right\}_{i}$ is unconditional, then we can assume without loss of generality that the basis $\left\{e_{i}\right\}_{i}$ is 1-unconditional in both Banach spaces $\left(X,\|\cdot\|_{X}\right)$ and $\left(X,\|\cdot\|_{1}\right)$ (see Remark 2.5 and Theorem 3.5). This implies that $\|\cdot\|_{X, 1}=\|\cdot\|_{1}$.

\section{THE FIRST KIND OF RENORMINGS}

In this section we show that some separable Banach spaces admit such renormings that the renormed Banach spaces have the fixed point property and at the same time they have some "bad" and some "good" geometrical properties. It is worth pointing out that the renormings used in this section were introduced in [5]. Moreover, this kind of renormings comes from the Karlovitz paper [16].

Let $(X,\|\cdot\|)$ be a Banach space with the Schauder basis $\left\{e_{i}\right\}_{i}$ and let $0<\beta<1$. We define a new norm $\|\cdot\|_{X, \beta}$ in $X$ as follows

$$
\|x\|_{X, \beta}:=\max \left\{\beta \sup _{k=0,1, \ldots}\left\|x-P_{k} x\right\|, \sup _{i=1,2, \ldots}\left|e_{i}^{*}(x)\right|\right\} .
$$

The norm $\|\cdot\|_{X, \beta}$ is equivalent to the original $\|\cdot\|([5])$.

In [5], the following theorems were proved.

Theorem 4.1. Let $(X,\|\cdot\|)$ be a Banach space with a Schauder basis. Then the Banach space $\left(X,\|\cdot\|_{X, \beta}\right)$ has the nonstrict Opial property. 
Theorem 4.2. Let $(X,\|\cdot\|)$ be a Banach space with a normalized Schauder basis. Then for $0<\beta \leq \frac{1}{2}$ the Banach space $\left(X,\|\cdot\|_{X, \beta}\right)$ lacks normal structure.

Now we can state the crucial result in this section.

Theorem 4.3. Let $(X,\|\cdot\|)$ be a Banach space with a 1 - unconditional and normalized Schauder basis $\left\{e_{i}\right\}_{i}$. Then we have

(1) the basis $\left\{e_{i}\right\}_{i}$ is 1 - unconditional in $\left(X,\|\cdot\|_{X, \beta}\right)$,

(2) $\left(X,\|\cdot\|_{X, \beta}\right)$ has the fixed point property for nonexpansive mappings,

(3) $\left(X,\|\cdot\|_{X, \beta}\right)$ has the non-strict Opial property,

(4) $\left(X,\|\cdot\|_{X, \beta}\right)$ does not have normal structure for $0<\beta \leq \frac{1}{2}$,

(5) $\left(X,\|\cdot\|_{X, \beta}\right)$ does not have asymptotic normal structure for $0<\beta \leq \frac{1}{4}$.

Proof. Obviously the basis $\left\{e_{i}\right\}_{i}$ is 1 - unconditional in $\left(X,\|\cdot\|_{X, \beta}\right)$ (see Remark 2.2). Therefore by the Lin Theorem (Theorem 2.7) the Banach space $\left(X,\|\cdot\|_{X, \beta}\right)$ has the fixed point property for nonexpansive mappings. It follows from Theorems 4.1 and 4.2 that the Banach space has the non-strict Opial property and for $0<\beta \leq \frac{1}{2}$ lacks normal structure.

Finally, the method of S. A. Mariadoss and P. M. Soardi [23] implies that the Banach space $\left(X,\|\cdot\|_{X, \beta}\right)$ with $0<\beta \leq \frac{1}{4}$ does not have asymptotic normal structure (see also the proof of Theorem 5.6 in Section $5)$.

Now observe that our considerations can be summarized in the following theorem.

Theorem 4.4. Let $(X,\|\cdot\|)$ be a Banach space with an unconditional basis. Then there exists an equivalent norm $\|\cdot\|_{0}$ such that

(1) $\left(X,\|\cdot\|_{0}\right)$ has the fixed point property for nonexpansive mappings,

(2) $\left(X,\|\cdot\|_{0}\right)$ has the non-strict Opial property,

(3) $\left(X,\|\cdot\|_{0}\right)$ fails to have asymptotic normal structure.

Proof. As we know there exists an equivalent norm $\|\cdot\|_{I}$ in $X$ such that the unconditional basis $\left\{e_{i}\right\}_{i}$ is 1-unconditional in $\left(X,\|\cdot\|_{I}\right)$ (see Remark 2.5). Additionally, by Remark 2.4, we can assume without loss of generality that our basis $\left\{e_{i}\right\}_{i}$ is normalized in $\left(X,\|\cdot\|_{I}\right)$. Now it is sufficient to apply Theorem 4.3 to the Banach space $\left(X,\|\cdot\|_{I}\right)$ to get the claimed norm $\|\cdot\|_{0}$.

\section{THE DAY NORM AND THE SECOND KIND OF RENORMINGS}

In this section we show another method of suitable renorming in a Banach space with the Schauder basis. This method is based on the Day norm in $c_{0}$.

We begin with the definition of the Day norm $\|\cdot\| \|$ in $c_{0}$ ([8], see also [22], [25] and [28]). If $u=\left\{u^{i}\right\} \in c_{0}$, then we enumerate the support of $u$ as $\{\tau(p)\}_{p}$ in such a way that $\left|u^{\tau(p)}\right| \geq\left|u^{\tau(p+1)}\right|$, define $D(u))=\left\{D^{i}(u)\right\} \in l^{2}$ by

$$
D^{i}(u)= \begin{cases}\frac{u^{\tau(p)}}{2^{p}}, & \text { if } i=\tau(p) \text { for some } p \\ 0, & \text { otherwise }\end{cases}
$$


and set $\mid\|u\|\|=\| D(u) \|_{2}$, where $\|\cdot\|_{2}$ is the standard norm in $l^{2}$. It is easy to observe that

$$
\frac{1}{2}\|u\|_{c_{0}} \leq\|\| u\left\|\mid \leq \frac{1}{\sqrt{3}}\right\| u \|_{c_{0}}
$$

for each $u \in c_{0}$, where $\|\cdot\|_{c_{0}}$ is the standard norm in $c_{0}$.

Remark 5.1. If $u=\left\{u^{i}\right\}_{i} \in c_{0}, j \in \mathbb{N}, a, b \in \mathbb{R},|a| \leq\left|u^{j}\right| \leq|b|$, and sequences $v=\left\{v^{i}\right\}_{i}, w=\left\{w^{i}\right\}_{i} \in c_{0}$ are such that

$$
v^{i}= \begin{cases}u^{i}, & \text { if } i \neq j, \\ a, & \text { if } i=j\end{cases}
$$

and

$$
w^{i}= \begin{cases}u^{i}, & \text { if } i \neq j \\ b, & \text { if } i=j\end{cases}
$$

then

$$
\||v|\| \leq\||u\||| \leq\|| w \mid\|
$$

(see [6], [8] and [25]).

Now let $\left\{e_{i}\right\}_{i}$ be a normalized Schauder basis in $(X,\|\cdot\|)$ and $\left\{e_{i}^{*}\right\}_{i}$ a sequence of biorthogonal functionals associated with this basis. As we know (see Remark 2.1) there is a constant $\tilde{M}_{1} \geq 1$ such that $\left\|e_{i}^{*}\right\| \leq \tilde{M}_{1}$ for each $i \in \mathbb{N}$. Fix $\alpha \in(0,1)$. It is easy to observe that for each $x \in X$ the sequence given by

$$
u(x)=\left\{u^{i}(x)\right\}_{i}=\left\{\alpha\|x\|, e_{1}^{*}(x), e_{2}^{*}(x), e_{2}^{*}(x), \ldots, e_{j}^{*}(x), \ldots, e_{j}^{*}(x), \ldots\right\}
$$

is an element of $c_{0}$ (here the $j$-th coordinate of $x$ is repeated exactly $j$ times). Now we use the Day norm $\||| \cdot||$ to the element $u(x)$ and set

$$
\|x\|_{L, \alpha}:=|\|u(x)\|| \mid=\|D(u(x))\|_{2},
$$

where $\|\cdot\|_{2}$ is the standard norm in $l^{2}$ (see [6] and [27]).

Remark 5.2. Obviously $\|\cdot\|_{L, \alpha}$ is a new norm in $X$. It is also easy to observe that

$$
\frac{\alpha}{2}\|x\| \leq\|x\|_{L, \alpha}=\|\| u(x) \mid\left\|\leq \frac{\tilde{M}_{1}}{\sqrt{3}}\right\| x \| .
$$

So the norm $\|\cdot\|_{L, \alpha}$ is equivalent to the norm $\|\cdot\|$ (see [6] and [27]).

In [6], the following results were proved.

Theorem 5.1. Let $(X,\|\cdot\|)$ be a Banach space with a Schauder basis. If $(X,\|\cdot\|)$ has the Kadec-Klee property, then the Banach space $\left(X,\|\cdot\|_{L, \alpha}\right)$ has the Kadec-Klee property.

Theorem 5.2. Let $(X,\|\cdot\|)$ be a Banach space with a Schauder basis. If $(X,\|\cdot\|)$ has the non-strict Opial property, then the Banach space $\left(X,\|\cdot\|_{L, \alpha}\right)$ has the non-strict Opial property.

Theorem 5.3. Let $(X,\|\cdot\|)$ be a Banach space with a normalized Schauder basis. If $0<\alpha \leq \frac{1}{2}$, then the Banach space $\left(X,\|\cdot\|_{L, \alpha}\right)$ lacks normal structure.

Theorem 5.4. Let $(X,\|\cdot\|)$ be a Banach space with a Schauder basis. Assume additionally that the Banach space $(X,\|\cdot\|)$ is reflexive and has the Kadec-Klee property. Then $\left(X,\|\cdot\|_{L, \alpha}\right)$ is LUR. 
We also have

Theorem 5.5. Let $(X,\|\cdot\|)$ be a Banach space with a 1 - unconditional Schauder basis $\left\{e_{i}\right\}_{i}$. Then the basis $\left\{e_{i}\right\}_{i}$ also is $1-$ unconditional in $\left(X,\|\cdot\|_{L, \alpha}\right)$.

Proof. If $\sum_{i=1}^{\infty} x^{i} e_{i}$ is a convergent series in $(X,\|\cdot\|)$, then we have

$$
\begin{gathered}
\left\|\sum_{i=1}^{\infty} x^{i} e_{i}\right\|=\left\|\sum_{i=1}^{\infty} \varepsilon_{i} x^{i} e_{i}\right\| \\
\left\{\left|u^{i}\left(\sum_{i=1}^{\infty} x^{i} e_{i}\right)\right|\right\}_{i}=\left\{\alpha\left\|\sum_{i=1}^{\infty} e_{i}^{*}(x) e_{i}\right\|,\left|x^{1}\right|,\left|x^{2}\right|,\left|x^{2}\right|, \ldots,\left|x^{j}\right|, \ldots,\left|x^{j}\right|, \ldots\right\}
\end{gathered}
$$

and

$$
\begin{gathered}
\left\{\alpha\left\|\sum_{i=1}^{\infty} \varepsilon_{i} e_{i}^{*}(x) e_{i}\right\|,\left|\varepsilon_{1} x^{1}\right|,\left|\varepsilon_{2} x^{2}\right|,\left|\varepsilon_{2} x^{2}\right|, \ldots,\left|\varepsilon_{j} x^{j}\right|, \ldots,\left|\varepsilon_{j} x^{j}\right|, \ldots\right\} \\
=\left\{\mid u^{i}\left(\sum_{i=1}^{\infty} \varepsilon_{i} x_{i} e_{i} \mid\right\}_{i}\right.
\end{gathered}
$$

Therefore

$$
\left\|\sum_{i=1}^{\infty} x^{i} e_{i}\right\|_{L, \alpha}=\left\|\sum_{i=1}^{\infty} \varepsilon_{i} x_{i} e_{i}\right\|_{L, \alpha}
$$

Now we are ready to prove two main theorems of this section.

Theorem 5.6. Let $(X,\|\cdot\|)$ be a Banach space with a 1-unconditional and normalized Schauder basis $\left\{e_{i}\right\}_{i}$. Assume $\|\cdot\|_{L, \alpha}$ is the defined above norm in $X$ with $0<\alpha<1$. Then

(1) the basis $\left\{e_{i}\right\}_{i}$ is 1 - unconditional in $\left(X,\|\cdot\|_{L, \alpha}\right)$,

(2) $\left(X,\|\cdot\|_{L, \alpha}\right)$ has the fixed point property for nonexpansive mappings,

(3) $\left(X,\|\cdot\|_{L, \alpha}\right)$ has the Kadec-Klee property under the additional assumption that $(X,\|\cdot\|)$ has the Kadec-Klee property,

(4) $\left(X,\|\cdot\|_{L, \alpha}\right)$ has the non-strict Opial property under the additional assumption that $(X,\|\cdot\|)$ has the non-strict Opial property,

(5) $\left(X,\|\cdot\|_{L, \alpha}\right)$ is LUR under the additional assumptions that $(X,\|\cdot\|)$ is reflexive and has the KadecKlee property,

(6) $\left(X,\|\cdot\|_{L, \alpha}\right)$ does not have normal structure for $0<\alpha \leq \frac{1}{2}$,

(7) $\left(X,\|\cdot\|_{L, \alpha}\right)$ does not have asymptotic normal structure for $0<\alpha \leq \frac{1}{4}$.

Proof. Obviously the basis $\left\{e_{i}\right\}_{i}$ is 1 - unconditional in $\left(X,\|\cdot\|_{L, \alpha}\right)$ (see Theorem 5.5). Therefore by the Lin Theorem (Theorem 2.7) the Banach space $\left(X,\|\cdot\|_{L, \alpha}\right)$ has the fixed point property for nonexpansive, mappings. It is sufficient to apply Theorem 5.1 to get the property $(3)$, i.e., $\left(X,\|\cdot\|_{L, \alpha}\right)$ has the KadecKlee property if $(X,\|\cdot\|)$ has this property.

Next, by Theorem 5.2, the Banach space $\|\cdot\|_{L, \alpha}$ has the non-strict Opial property if $(X,\|\cdot\|)$ has this property. By Theorem 5.3, $\left(X,\|\cdot\|_{L, \alpha}\right)$ does not have normal structure for $0<\alpha \leq \frac{1}{2}$. By Theorem 5.4, if $(X,\|\cdot\|)$ is reflexive and has the Kadec-Klee property, then $\left(X,\|\cdot\|_{L, \alpha}\right)$ is LUR. 
Finally, as in [23] (see also [2]), we set

$$
x_{n}= \begin{cases}\left(1-\frac{j}{2^{2 i}}\right) e_{i}+e_{i+1}, & \text { if } n=2^{2 i}+j, \quad j=1,2, \ldots, 2^{2 i} \\ e_{i+1}+\frac{j}{2^{2 i+1}} e_{i+2}, & \text { if } n=2^{2 i+1}+j, \quad j=1,2, \ldots, 2^{2 i+1} .\end{cases}
$$

and

$$
C=\overline{\operatorname{conv}}\left\{x_{n} ; n=5,6, \ldots\right\}
$$

Then for $0<\alpha \leq \frac{1}{4}$ we have

$$
0=\lim _{n}\left\|x_{n}-x_{n+1}\right\|=\lim _{n}\left\|x_{n}-x_{n+1}\right\|_{L, \alpha}
$$

and

$$
\operatorname{diam}_{\|\cdot\|}(C) \leq 4
$$

for the diameter of $C$ in the norm $\|\cdot\|$. Hence directly from the definition of the norm $\|\cdot\|_{L, \alpha}$ (see also Remark 5.1) we get

$$
\left\|x_{n_{2}}-x_{n_{1}}\right\|_{L, \alpha} \leq\left[\sum_{k=1}^{\infty} 4^{-k}\right]^{\frac{1}{2}}=\frac{1}{\sqrt{3}}
$$

for each $n_{1}, n_{2} \in \mathbb{N}$. Next for each $\tilde{n} \in \mathbb{N}$ we have

$$
\lim _{n}\left\|x_{n}-x_{\tilde{n}}\right\|_{L, \alpha}=\left[\sum_{k=1}^{\infty} 4^{-k}\right]^{\frac{1}{2}}=\frac{1}{\sqrt{3}}
$$

and in a similar way we obtain

$$
\left\|\sum_{l=1}^{m} a_{l} x_{l}-x_{n}\right\|_{L, \alpha} \geq\left[\sum_{k=1}^{i+1} 4^{-k}\right]^{\frac{1}{2}}
$$

for each convex combination $\sum_{l=1}^{m} a_{l} x_{l}$ and for every sufficiently large $n$, where $i$ is connected with $x_{n}$ by $(*)$. This implies

$$
\operatorname{diam}_{\|\cdot\|_{L, \alpha}}(C)=\operatorname{diam}_{\|\cdot\|_{L, \alpha}}\left\{x_{n}\right\}=\frac{1}{\sqrt{3}}
$$

and

$$
\lim _{n}\left\|x-x_{n}\right\|_{L, \alpha}=\operatorname{diam}_{\|\cdot\|_{L, \alpha}}(C)
$$

for each $x \in C$ and therefore the set $C$ lacks asymptotic normal structure.

Remark 5.3. A generalization $\|\cdot\|_{\beta, p}$ of the Day norm was introduced in [3] and Theorem 5.6 is also valid for the norm in $X$ generated by $\|\cdot\|_{\beta, p}$ analogously as the norm $\|\cdot\|_{L, \alpha}$ in $l^{2}$ (see [4]).

We summarize our considerations in the final result of this paper.

Theorem 5.7. Let $(X,\|\cdot\|)$ be a Banach space with an unconditional Schauder basis. Then there exists an equivalent norm $\|\cdot\|_{00}$ such that

(1) $\left(X,\|\cdot\|_{00}\right)$ has the fixed point property for nonexpansive mappings,

(2) $\left(X,\|\cdot\|_{00}\right)$ has the Kadec-Klee property,

(3) $\left(X,\|\cdot\|_{00}\right)$ has the non-strict Opial property,

(4) $\left(X,\|\cdot\|_{00}\right)$ is LUR under the additional assumption that $(X,\|\cdot\|)$ is reflexive,

(5) $\left(X,\|\cdot\|_{00}\right)$ does not have asymptotic normal structure.

Proof. It is sufficient to apply Remark 2.5 and Theorems 3.6 and 5.6. 


\section{Acknowledgements}

These results have been partially achieved within the framework of the STREVCOMS Project No. 612669 with funding from the IRSES Scheme of the FP7 Programme of the European Union (the first author and the fourth author).

\section{REFERENCES}

[1] A .G. Aksoy, M. A. Khamsi, Nonstandard Methods in Fixed Point Theory, Springer-Verlag, 1990.

[2] J.-B. Baillon, R. Schöneberg, Asymptotic normal structure and fixed points of nonexpansive mappings, Proc. Amer. Math. Soc. 81 (1981), 257-264.

[3] M. Budzyńska, A. Grzesik, M. Kot, The generalized Day norm. Part I. Properties, Ann. Univ. Mariae Curie-Skłodowska Sect. A, 71 (2017), 33-49.

[4] M. Budzyńska, A. Grzesik, M. Kot, The generalized Day norm. Part II. Applications, Ann. Univ. Mariae CurieSkłodowska Sect. A, 71 (2017) 51-62.

[5] M. Budzyńska, A. Grzesik, W. Kaczor, T. Kuczumow, Schauder bases and diametrically complete sets with empty interior, preprint.

[6] M. Budzyńska, W. Kaczor, M. Kot, T. Kuczumow, Schauder bases, LUR and diametrically complete sets with empty interior, preprint.

[7] W. J. Davis, W. B. Johnson, A renorming of nonreflexive Banach spaces, Proc. Amer. Math. Soc. 37 (1973), $486-488$.

[8] M. M. Day, Strict convexity and smoothness of normed spaces, Trans. Amer. Math. Soc. 78 (1955), 516-528.

[9] P.G. Dodds, T. K. Dodds, A. A. Sedaev, F. A. Sukochev, Local uniform convexity and Kadec-Klee type properties in K-interpolation spaces. I: General theory, J. Funct. Spaces Appl. 2 (2004), 125-173.

[10] T. Domínguez Benavides, A renorming of some nonseparable Banach spaces with the fixed point property, J. Math. Anal. Appl. 350 (2009), 525-530.

[11] D. van Dulst, Equivalent norms and fixed point property for nonexpansive mappings, J. London Math. Soc. 25 (1982), 139-144.

[12] K. Goebel, W. A. Kirk, Topics in Metric Fixed Point Theory, Cambridge University Press, 1990.

[13] K. Goebel, S. Reich, Uniform Convexity, Hyperbolic Geometry, and Nonexpansive Mappings, Marcel Dekker, 1984.

[14] R. B. Holmes, Geometric Functional Analysis and its Applications, Springer-Verlag, 1975.

[15] M. I. Kadec, On the connection between weak and strong convergence, Dopovidi Akad. Nauk Uktain. RSR 9 (1959), 949-952.

[16] L. A. Karlovitz, Existence of fixed points of nonexpansive mappings in a space without normal structure, Pacific J. Math. 66 (1976), 153-159.

[17] W. A. Kirk, A fixed point theorem for mappings which do not increase distances, Amer. Math. Monthly 72 (1965), 1001006.

[18] V. Klee, Mappings into normed linear spaces, Fund. Math. 49 (1960/1961), 25-34.

[19] P.-K. Lin, Unconditional bases and fixed points of nonexpansive mappings, Pacific J. Math 116 (1985), 69-76.

[20] J. Lindenstrauss, L. Tzafriri, Classical Banach Spaces I and II, Springer, 1977.

[21] A. R. Lovaglia, Locally uniformly convex Banach spaces, Trans. Amer Math. Soc. 78 (1955), 225-238.

[22] E. Maluta, A diametrically complete set with empty interior in a reflexive LUR space, J. Nonlinear Convex Anal. 18 (2017), 105-111.

[23] S. A. Mariadoss, P. M. Soardi, A remark on asymptotic normal structure in Banach spaces, Rend. Sem. Mat. Univ. Politec. Torino 44 (1986), 393-395.

[24] Z. Opial, Weak convergence of the sequence of successive approximations for nonexpansive mappings, Bull. Amer. Math. Soc. 73 (1967), 591-597.

[25] J. Rainwater, Local uniform convexity of Day's norm on $c_{0}(\Gamma)$, Proc. Amer. Math. Soc. 22 (1969), 335-339.

[26] I. Singer, Bases in Banach Spaces I, Springer, 1970).

[27] M. A. Smith, Some examples concerning rotundity in Banach spaces, Math. Ann. 233 (1978), 155-161. 
[28] M. A. Smith, B. Turett, A reflexive LUR Banach spaces that lacks normal structure, Canada Math. Bull. 28 (1985), 492-494.

[29] V. Zizler, Some notes on various rotundity and smoothness properties of separable Banach spaces, Comment. Math. Univ. Carolinae 10 (1969), 195-206. 